\title{
Untersuchung von Biofilmen mit dem Rasterkraftmikroskop
}

\author{
Prof. Dr. rer. nat. Asta Richter, Prof. Dr. Franz Xaver Wildenauer, Dipl.-Kristallogr. Ronald Ries
}

\section{Einleitung}

Technische Prozesse können erheblich durch Biofilme beeinflußt werden und spielen in weiten Bereichen der Technik und Biotechnologie eine große Rolle /1, 2/. Ein zentrales Problem beim Einsatz von Werkstoffen in biologischen Systemen ist die Ansiedlung von Zellen und deren Stoffwechselprodukten auf Festkörperoberflächen. Erst hiervon gehen alle nachfolgenden Reaktionen aus, wie Kontamination von Implantaten und medizinischen Geräten, Biokorrosion und die Bildung von Biofilmen in technischen Geräten. Technische Prozesse können erheblich durch Biofilme beeinflußt werden und spielen in weiten Bereichen der Technik und Biotechnologie eine große Rolle.

Die Fähigkeit, die unterschiedlichsten Oberflächen und Materialien rasch zu besiedeln, bietet vielen Mikroorganismen einen erheblichen Selektionsvorteil, weil sie sich permanent unmittelbar in der Nähe einer Nährstoffquelle aufhalten können und nicht mit dem strömenden Medium weiter transportiert werden. Dabei spielt die Anwesenheit von Sauerstoff keine Rolle. Biofilme werden technisch vor allem in der Umwelttechnik zur Reinigung von Abwasser und Abluft genutzt. Dabei kommen vor allem Kunststoffoberflächen in Form von Ringen oder Wellplatten aus PVC oder PP zum Einsatz. In der Biotechnologie wird dagegen bevorzugt poröses Glas zum Ruickhalt von Mikroorganismen oder als Aufwuchsmaterial für tierische oder pflanzliche Zellen genutzt. In beiden Fällen wird erreicht, daß die Aufenthaltszeit der Mikroorganismen von der Verweilzeit des Mediums im Reaktor entkoppelt wird.

Unerwünscht ist dagegen die Bildung von Biofilmen beim Einsatz von Werkstoffen in biologischen Systemen und in der Hygienetechnik. Eignungsuntersuchungen und qualitative Überprüfung von mikrobiellem Bewuchs an Oberflächen erfolgen bislang meist durch einfache Lichtmikroskopie von besiedelten Objektträgern in den unterschiedlichsten Medien oder durch Untersuchung von abgeschabtem Material (Plaques), von abgelösten Flocken und Zellverbänden oder durch Entnahme von Trägermaterial und Überprüfung im Rasterelektronenmikroskop. Die häufig gefundene Polymermatrix aus Kohlenhydraten oder Protein-Präzipitaten der Biofilme adsorbiert vor allem Nährstoffe für das Wachstum der fixierten Organismen und schützt sie zugleich vor Angriffen durch das Immunsystem. Diese Tatsache erschwert den Einsatz von künstlichen medizinischen Implantaten, die später als Oberfläche für die Bildung von pathogenen Biofilmen dienen können. In der Verfahrenstechnik können Biofilme Oxidations- oder Reduktionsreaktionen (Biokorrosion) auslösen oder die Funktion von technischen Anlagen oder Apparaten bis hin zu vollständigen Verstopfungen beeinträchtigen (Biofouling).
Die in der Biotechnologie eingesetzten Werkstoffe müssen bestimmten technischen und biologischen Forderungen genügen. Die Klärung der Adhäsionsmechanismen der Bakterien auf unterschiedlichen Werkstoffoberflächen und des sich aus einem ersten Zellhaufen entwickelnden Biofilms sowie die Reaktionen und die Transportmechanismen an und in ihm sind die Voraussetzung zur Interpretation der Werkstoffschädigung und des Zellverhaltens. Neben der konventionellen Licht- und Rasterelektronenmikroskopie steht mit der Raster-Kraft-Mikroskopie (SFM) ein neues Verfahren zur Abbildung von Oberflächen biologischer Systeme mit hoher Auflösung /3 -5/ zur Verfügung. Ziel der Arbeiten ist es, mit dem SFM Untersuchungen von Biofilmen auf verschiedenen Werkstoffoberflächen durchzuführen. Hierzu ist es notwendig, die Raster-Kraft-Mikroskopie an die Besonderheiten biologischer Strukturen anzupassen. Aufgrund dieser Oberflächenuntersuchungen lassen sich dann geeignete Werkstoffe und Beschichtungen fuir den Einsatz in biologischen Systemen auswählen.

\section{Wachstum der Biofilme}

In wäßrigen Nährmedien wurden mit verschiedenen Bakterienarten mikrobielle Filme auf unterschiedlichen Materialien wie Dickglas, Keramik, Silizium und Molybdänblech erzeugt. Auf diesen Materialien wurden gezielt Biofilme mit Stäbchenbakterien und Purpur-Schwefelbakterien /6, 7/ aufgewachsen. Die Mikroorganismen Bacillus subtilis sporum sind ein gram positives, aerobes sporenbildendes Stäbchen. Die $250 \mathrm{ml}$ Erlenmeyerkolben wurden zunächst mit den unterschiedlichen Materialträgern bei $121^{\circ} \mathrm{C}$ autoklaviert und anschließend mit ca. $100 \mathrm{ml}$ DEV-Standard-Nährboullion befuillt. Sie wurden aus einer logarithmisch wachsenden Kultur auf eine Dichte von $108 \mathrm{Keime} / \mathrm{ml}$ beimpft. Anschließend wurde bei $37{ }^{\circ} \mathrm{C}$ stehend über $48 \mathrm{~h}$ inkubiert. Nachdem die Kolben dicht bewachsen waren, wurden die Materialträger entnommen und die Biofilme einer Gefriertrocknung unterzogen.

Zur Untersuchung der Bildung anaerober Biofilme aus Mischkulturen auf verschiedenen Trägern wurde eine Probe von kommunalem anaeroben Klärschlamm in ein 3 I Standgefäß gefüllt, luftdicht verschlossen und bei Raumtemperatur ca. $18-20{ }^{\circ} \mathrm{C}$ uiber 3 Monate stehengelassen. Das entstehende Biogas mit einem Methananteil von 50 bis $65 \%$ wurde aufgefangen und die Gasproduktion aufgezeichnet. Nach 3 Monaten kam die Gasproduktion praktisch zum erliegen. Es wurde eine dichte Besiedlung der Glasoberfläche über dem Schlammbett mit Purpur-Schwefelbakterien beobachtet. Die Aufwuchsträger waren so positioniert, daß sie nicht in 
das Sediment eintauchten, sondern sich in dem mehr oder weniger klaren Überstand befanden. Nach weiteren 4 Wochen wurden die Probenträger entnommen, mit einer Gefriertrocknung eine Fixierung durchgefiihrt und auf Bewuchs untersucht.

Das Wachstumsverhalten mikrobieller Beläge wurde mit Lichtmikroskopie und Raster-Kraft-Mikroskopie untersucht.

\section{Raster-Kraft-Mikroskopie (SFM)}

Die Abbildung kleiner Strukturen ist seit Jahrhunderten eine Herausforderung für den menschlichen Erkenntnisdrang. Wichtige Etappen der Entwicklung der Mikroskopie sind in Abb. 1 dargestellt. Die Anwendung von Vergrößerungsgläsern führte schließlich im 16. Jahrhundert zum Bau erster, aus zwei Linsen bestehender Lichtmikroskope. Bereits Leeuwenhoek nutzte dieses einfache optische Gerät zur Untersuchung von Bakterien und roten Blutkörperchen. Inzwischen hat die Lichtmikroskopie mit der Entwicklung von Polarisations- und Lasermikroskopen sowie mit der konfokalen Lichtmikroskopie eine enorme Verbesserung erfahren. Ein weiterer Meilenstein bei der Erforschung des Mikrokosmos ist die Anwendung der Elektronenstrahlen zur Abbildung kleinster Strukturen. Auflösung und Vergrößerung werden im Vergleich zur Lichtmikroskopie um Größenordnungen übertroffen. Damit sind Rasterelektronen- (SEM) und Transmissionselektronenmikroskopie (TEM) heutzutage bei der Untersuchung organischer und anorganischer Mikro- und Nanostrukturen wichtige Standardtechniken. Mit hochauflösenden Geräten lassen sich Details im Sub-Nanometerbereich nachweisen. Allerdings sind aufwendige Präparationstechniken für die Untersuchungen im Elektronenmikroskop notwendig. Lebende Objekte lassen sich mit der Elektronenmikroskopie nicht untersuchen.

Anfang der 80iger Jahre wurde mit dem Raster-TunnelMikroskop (STM) /8/ ein neues Prinzip der Oberflächenanalyse gefunden. Dabei wird die Oberfläche von Ma-

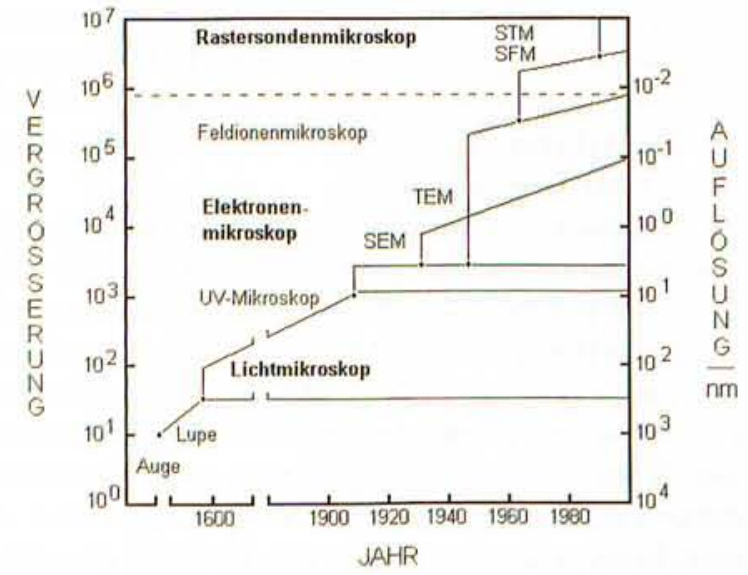

Abb. 1: Zeitliche Entwicklung der Mikroskopie mit Angabe der Vergrößerung und der Auflösung. terialien mit einer feinen, präzise gesteuerten nadelförmigen Spitze abgerastert. Der zwischen Spitze und Oberfläche fließende Tunnelstrom wird registriert und zur Abbildung der Oberflächenstruktur verwendet. Diese verbliiffende Idee der Ausnutzung von Quanteneffekten führt unter Einbeziehung anderer Wechselwirkungsmechanismen wie z. B. atomaren Wechselwirkungskräften, magnetischen und elektrochemischen Wechselwirkungen oder thermischen Feldern zu einer ganzen Familie sogenannter Rastersondenverfahren. Diese Oberflächenanalyseverfahren zeichnen sich durch eine hohe laterale Auflösung der Oberflächentopographie, geringen Präparationsaufwand und einfache Handhabung aus $/ 9,10 /$.

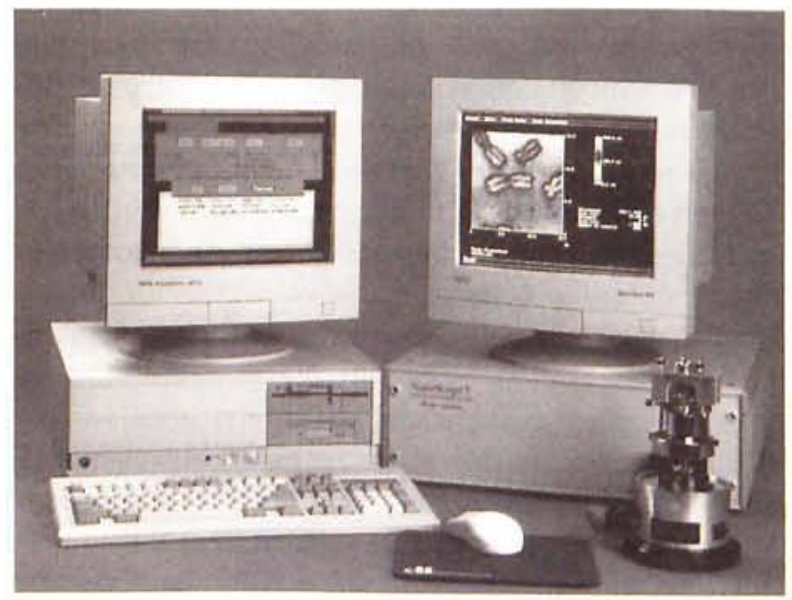

Abb. 2: Raster-Kraft-Mikroskop: (a) Geräteset

In Abb. 2 ist das Raster-Kraft-Mikroskop (SFM), ein Nanoskop der Firma digital instruments, wie es an der TFH Wildau verfügbar ist, dargestellt. Das Funktionsprinzip des SFM basiert auf der Registrierung der abstoßenden bzw. anziehenden Kräfte zwischen einer atomar feinen Sondenspitze und der Probenoberfläche, die dadurch entsteht, daß sich die Elektronenwolken beider Materialien überlappen. Die registrierten Wechselwirkungskräfte liegen im Bereich von $0,05 \mathrm{nN}$ bis $70 \mathrm{nN}$. Mit diesem Verfahren ist es möglich, sowohl elektrisch leitende als auch nichtleitende Proben zu untersuchen. Die Abtastung der Oberfläche kann in Luft, im Vakuum und in Fliissigkeiten erfolgen. Dies hat den Vorteil, daß aufwendige Präparationsarbeiten wie für das Elektronenmikroskop entfallen und auch lebende Objekte untersucht werden können. So können Materialoberflächen bis hin zu atomaren Details $/ 11$ / als auch komplexe biologische Systeme / $-5 /$ in ihrer physiologischen Umgebung abgebildet werden.

In Abb. 2 b ist das eigentliche Funktionsprinzip des SFM dargestellt. Ein Laserstrahl wird auf die Spitze des flexiblen Cantilevers fokussiert und auf eine zweigeteilte Fotodiode abgebildet. Die feine, pyramidenförmige Tastspitze (Abb. 2 c), die hier mit dem Rasterelektronenmikroskop abgebildet wurde, befindet sich an einem dünnen Hebelarm mit extrem kleiner Federkonstante und rastert die Probenoberfläche punktförmig ab. Verschiedene Materialien wie $\mathrm{Si}_{3} \mathrm{~N}_{4}$, Silizium- oder Diamanteinkristalle unterschiedlicher Form werden als Abt- 


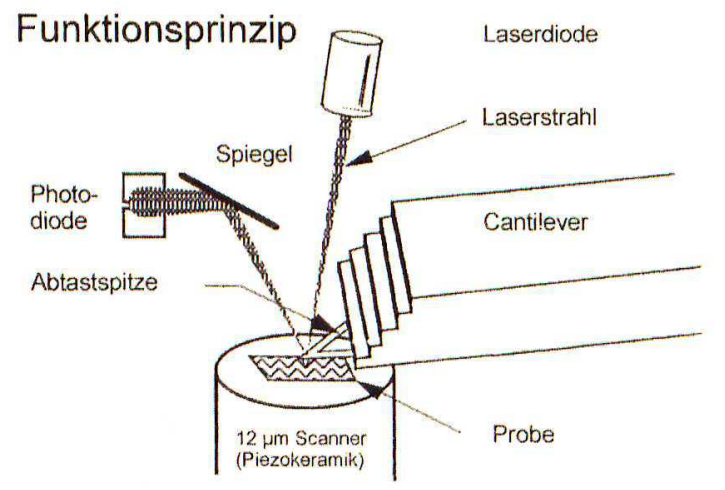

Abb. 2: Raster-KraftMikroskop: (b) Funktionsprinzip, (c) pyramidenförmige Spitze aus $\mathrm{Si}_{3} \mathrm{~N}_{4}$ aufgenommen im Rasterelektronenmikroskop bei 7.000 facher Vergrößerung.

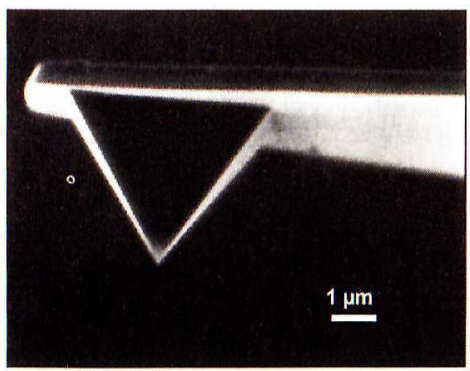

astspitzen verwendet. Über die Piezoaktuatoren wird die Auflagekraft der Spitze mit einem geeigneten Feedback so gesteuert, daß sie konstant bleibt. Auslenkungen der Spitze bzw. des Hebelarmes infolge des geometrischen Oberflächenprofils werden mit dem Abstandssensor als Änderungen registriert. Die durch das Oberflächenprofil verursachte Verbiegung des Cantilevers wird optisch nachgewiesen, wobei die Änderung der Position des Laserstrahls auf der Spitze durch die Spannungsdifferenz an der Fotodiode registriert wird. Aus diesen Meßdaten entsteht das dreidimensionale topographische Oberflächenprofil der Probe.

\section{Ergebnisse}

Die Bildung von Biofilmen auf unterschiedlichen Werkstoffen wurde mit dem Lichtmikroskop und dem SFM untersucht. Es zeigte sich, daß das Wachstumsverhalten der Mikroorganismen auf unterschiedlichen Werkstoffen verschieden ist. Die Bildung von Biofilmen mit den

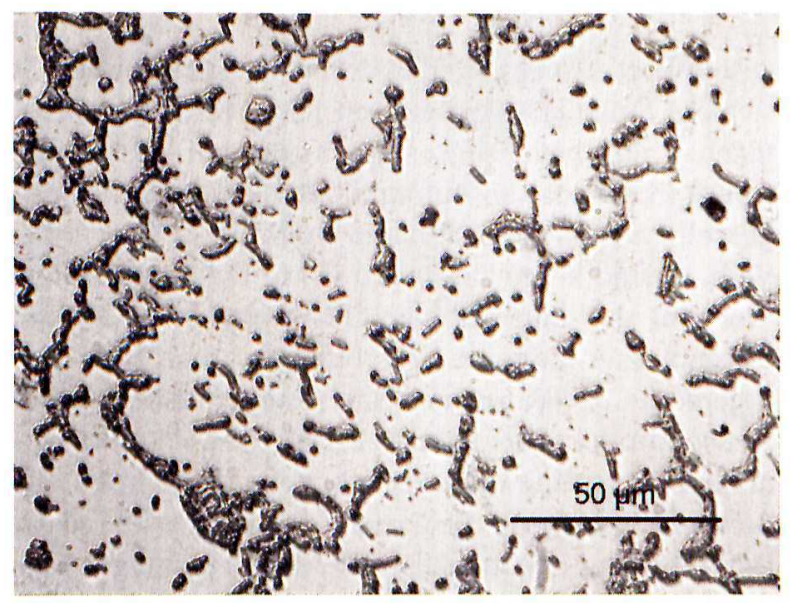

Abb. 3: Stäbchenbakterien auf Keramiksubstrat: (a) lichtmikroskopische Aufnahme

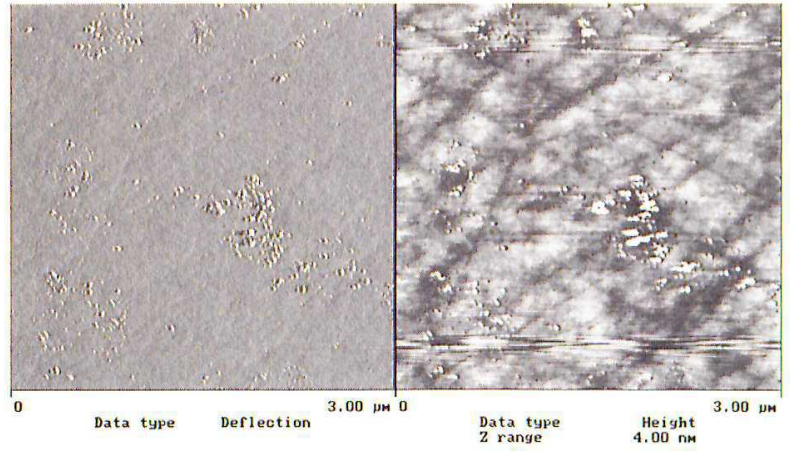

Abb. 3: Stäbchenbakterien auf Keramiksubstrat: (b) SFM-Bild der ursprünglichen Keramikoberfläche

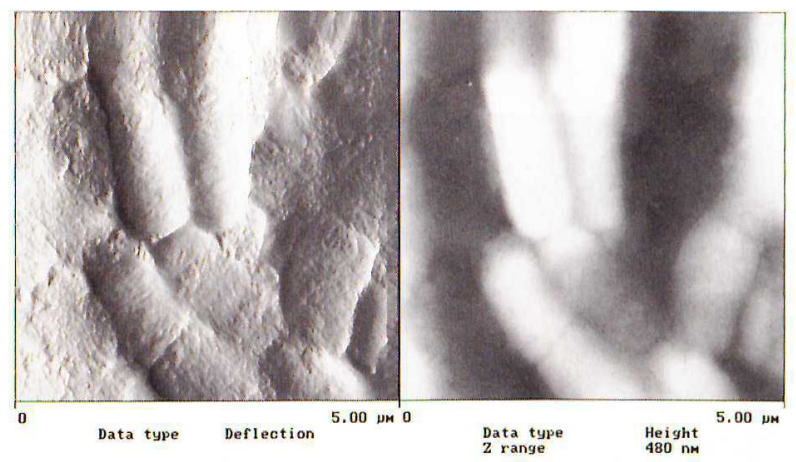

Abb. 3: Stäbchenbakterien auf Keramiksubstrat: (c) SFM-Bild des Mikroorganismen-Clusters

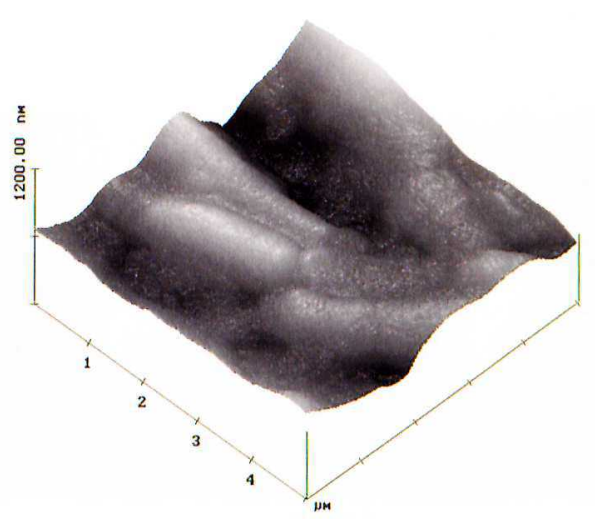

Abb. 3: Stäbchenbakterien auf Keramiksubstrat: (d) dreidimensionale SFMAbbildung der Bakterien

Mikroorganismen Bacillus subtilis sporum (Abb. $3-6$ ) zeigt deutlich ein Wachstum uiber Clusterbildung. Die lichtmikroskopischen Abbildungen machen deutlich, daß die Belegungsdichte der Substrate mit Biomaterial unterschiedlich ist. Auf dem Keramiksubstrat (Abb. 3) sind größere Zellhaufen sichtbar, die bereits teilweise in zusammenhängende Perkolationsstrukturen übergehen, während auf Dickglas (Abb. 4) kleinere Cluster deutlich sichtbar sind. Auf beiden Substraten, Dickglas und Keramik, sind einzelne Bakterienhaufen fest auf der Oberfläche verankert und gut im SFM sichtbar. Es scheint, daß die Mikroorganismen in eine Gel-Matrix eingebettet und immobilisiert sind. Die Bakterien haben erwartungsgemäß eine Länge von 2-3 $\mu \mathrm{m}$ und einen Durchmesser von $1 \mu \mathrm{m}$. Deutlich sind Einschniirungen bei der Zellteilung sichtbar. Die Oberfläche der Peptidoglycan-Huille ist erwartungsgemäß nur gering 


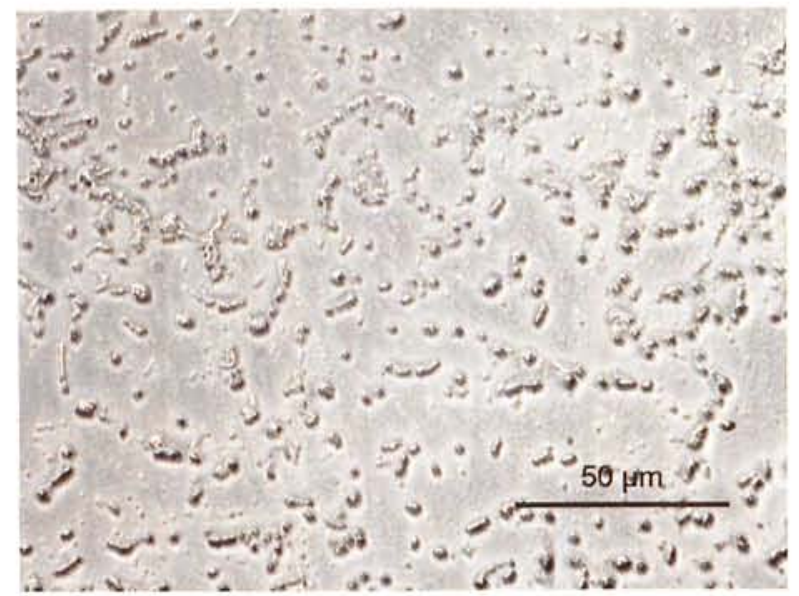

Abb. 4: Stäbchenbakterien auf Dickglassubstrat: (a) lichtmikroskopische Aufinalıme

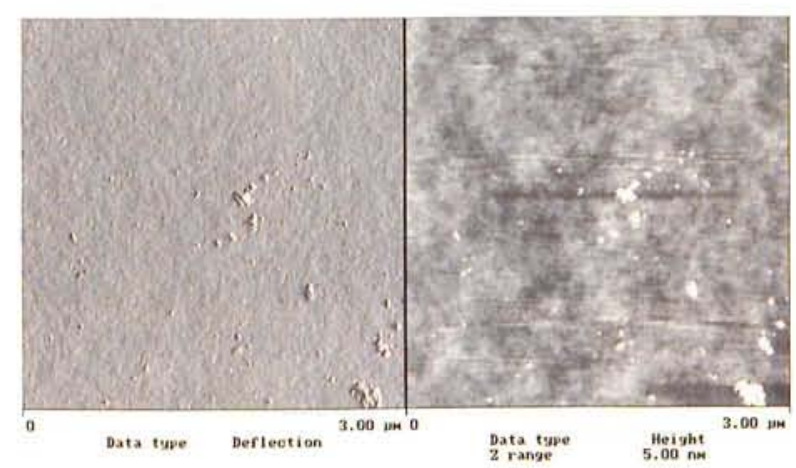

Abb. 4: Stäbchenbakterien auf Dickglassubstrat: (b) SFM-Bild der ursprünglichen Dickglasoberfläche

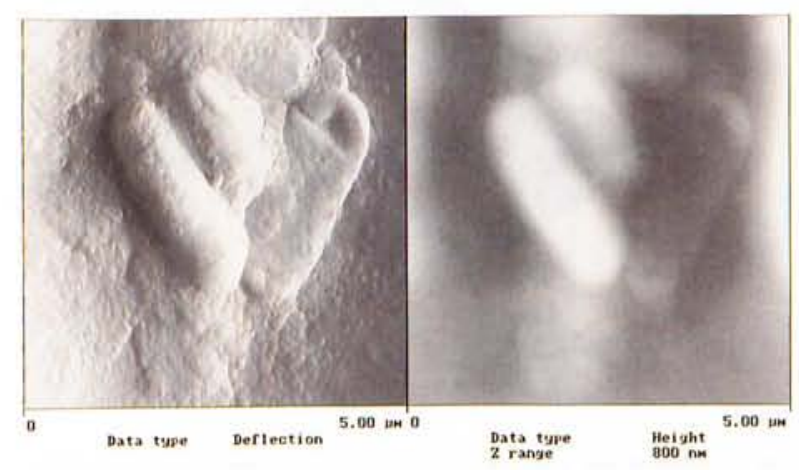

Abb. 4: Stäbchenbakterien auf Dickglassubstrat: (c) SFM-Bild des Mikroorganismen-Clusters

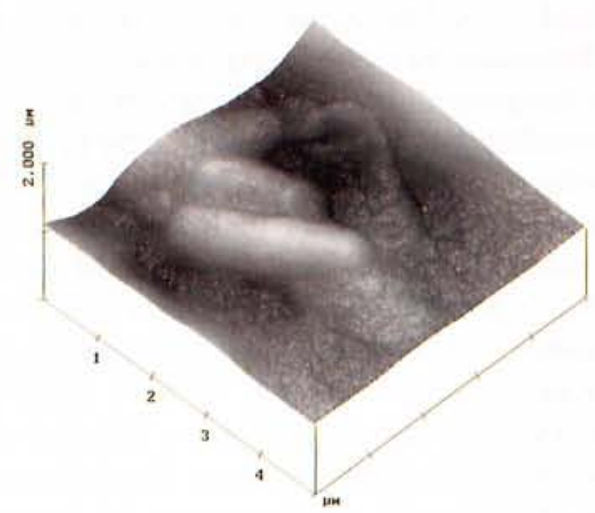

Abb. 4: Stäbchenbakterien auf Dickglassubstrat: (d) dreidimensionale SFMAbbildung der Bakterien

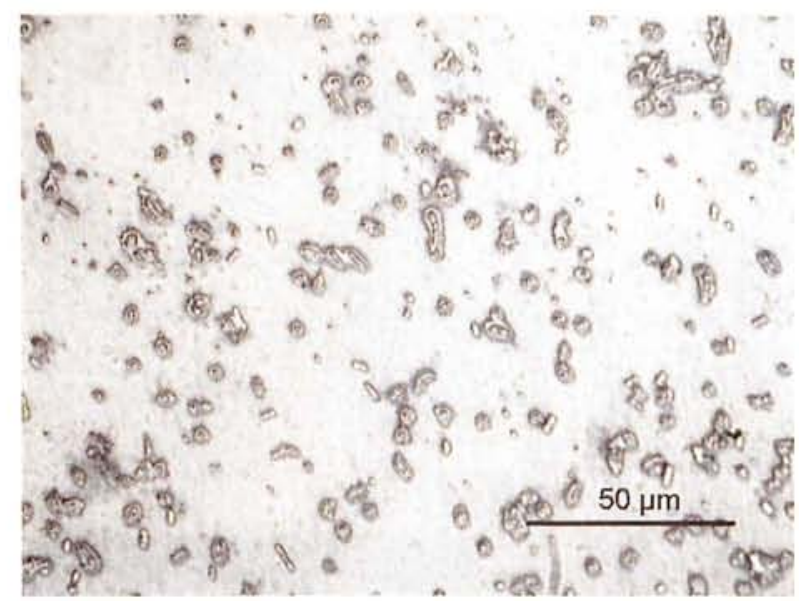

Abb. 5: Stäbchenbakterien auf Siliziumsubstrat: (a) lichtmikroskopische Aufnalume

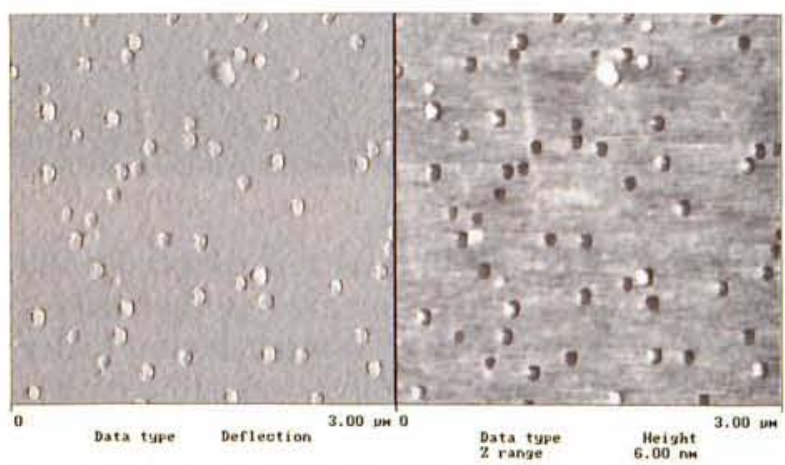

Abb. 5: Stäbchenbakterien auf Siliziumsubstrat: (b) SFM-Bild der ursprünglichen Si (100)-Oberfläche

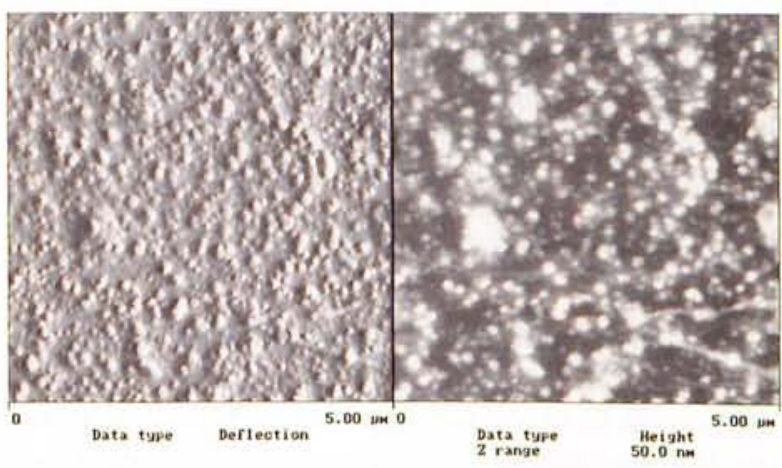

Abb. 5: Stäbchenbakterien auf Siliziumsubstrat: (c) SFM-Bild des Biofilms.

strukturiert. Mit der dreidimensionalen Darstellung läßt sich die räumliche Struktur der Bakterien gut sichtbar machen. Ein bevorzugtes Wachstum auf kristallinen Oberflächen oder an Aufrauhungen konnte nicht festgestellt werden. Auf Silizium (Abb.5) und Molybdän (Abb. 6) sind keine haftenden Bakterien zu erkennen. Vielmehr sind kugelförmige Cluster von $200-300 \mathrm{~nm}$ Durchmesser als feine Verteilung sichtbar. Dies sind organische Beläge aus der Nährlösung und/oder Stoffwechselprodukte der Bakterien. Haftende Mikroorganismen oder Proteine konnten nicht gefunden werden. Die Belegung der Oberfläche ist noch geringer als im Fall der Dickglas- bzw. Keramiksubstrate. Eine bevorzugte Lage der Cluster entlang kristallographischer Richtungen oder entlang von Walzrichtungen bzw. Riefen bei Molybdänblech konnte nicht gefunden werden. 


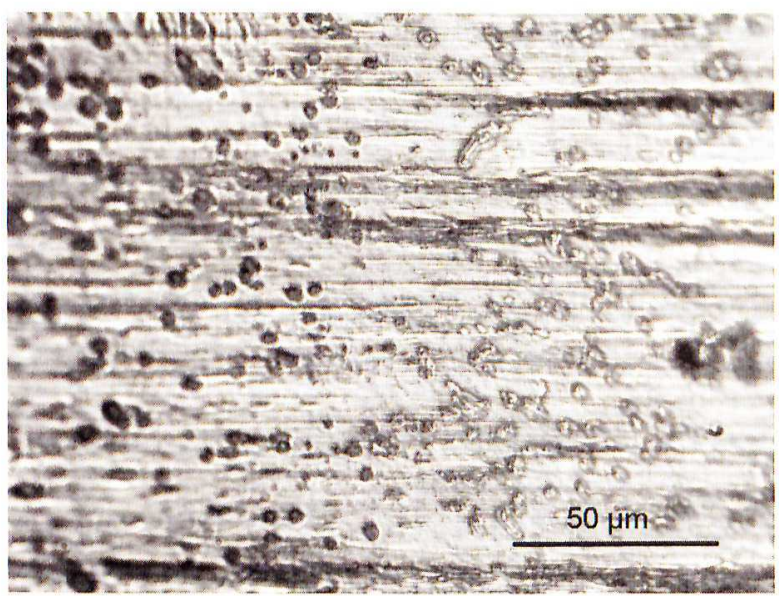

Abb. 6: Stäbchenbakterien auf Molybdänsubstrat: (a) lichtmikroskopische Aufnahme

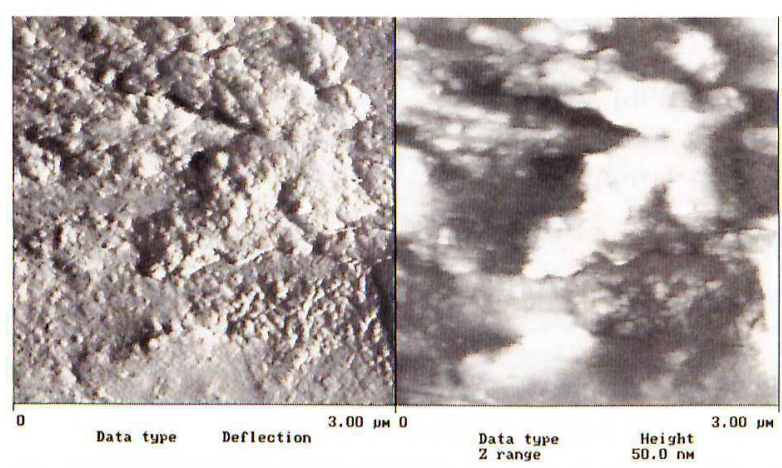

Abb. 6: Stäbchenbakterien auf Molybdänsubstrat: (b) SFM-Bild des Biofilms.

Im Gegensatz zu den Stäbchenbakterien wachsen die anaeroben Bakterien-Mischkulturen sehr gut auf Siliziumsubstraten auf (Abb.7). Cluster aus Mikroorganismen, die 2-3 $\mu \mathrm{m}$ lang sind und einen Durchmesser von 0,7-1 $\mu \mathrm{m}$ haben, sind deutlich sichtbar. Sogar geißelförmige Gebilde konnten gefunden werden. Neben haftenden, in Clustern angeordneten Purpur-Schwefelbakterien befinden sich Stoffwechselprodukte oder Proteinpräzipitate in lockerer Belegung auf der Oberfläche. Einige Mikroorganismen wurden bei der Präparation zerstört und sind ausgelaufen, so daß nur noch die eingefallenen Hiille sichtbar ist (Abb. 7 d). In Abb. 8 sind zum Vergleich noch einmal dreidimensionale Topographiebilder der beiden Bakteriensorten dargestellt. Die Höhenstruktur, aber auch Einzelheiten der Zellwand werden deutlich sichtbar.

Es wurde nachgewiesen, daß sich die SFM-Analysentechnik gut zur Untersuchung von Biofilmen eignet. Damit wurde eine neue Methode angewandt, um ein dreidimensionales Abbild der Oberfläche von biologischen Systemen in ihrer nativen Umgebung mit hoher Auflösung zu erhalten. Hieraus ergeben sich neue wissenschaftliche Aspekte im Vergleich zu anderen strukturellen Untersuchungsmethoden wie Röntgen- und Neutronenbeugung sowie Elektronenmikroskopie.

Für die Besiedlung der Werkstoffoberfläche mit Zellen ist sowohl das Material als auch die Oberflächenrauhigkeit entscheidend. Die Bakterienarten haben unterschiedliche Wechselwirkungsmechanismen mit ver-

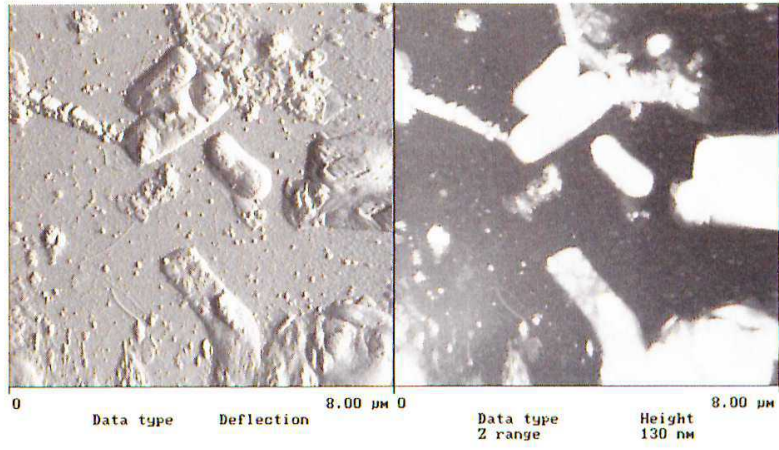

Abb. 7: Purpur-Schwefelbakterien auf Siliziumsubstrat: (a) -(d) SFM-Bilder des Biofilms mit Clustern aus Mikroorganismen bei verschiedenen Abbildungsgrößen.

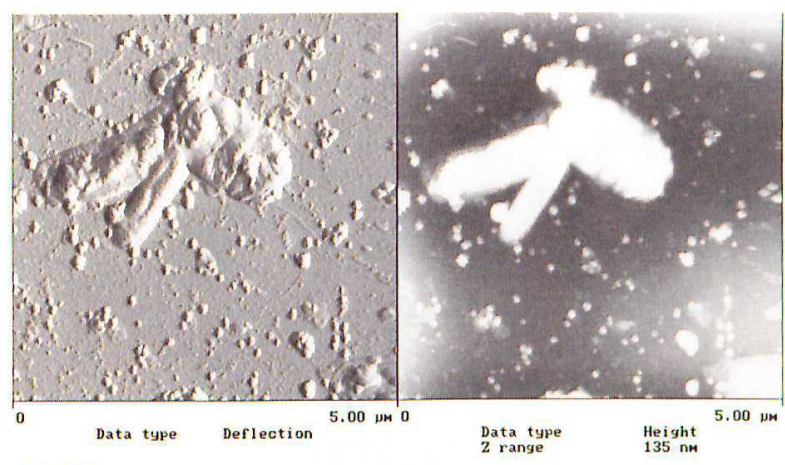

Abb. $7(b)$

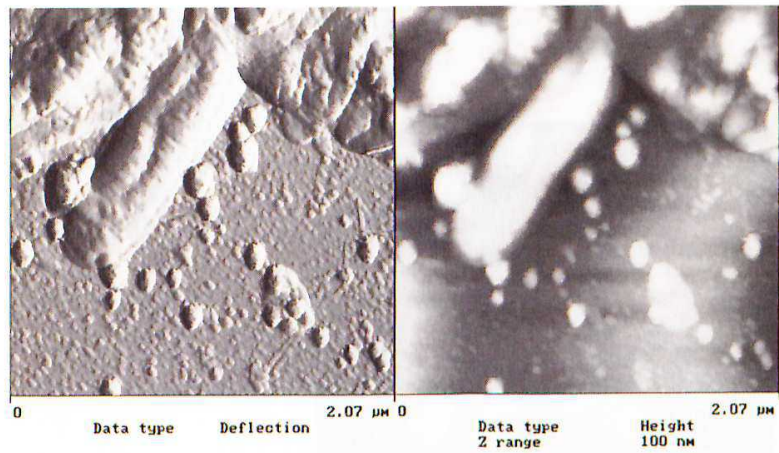

Abb. 7(c)

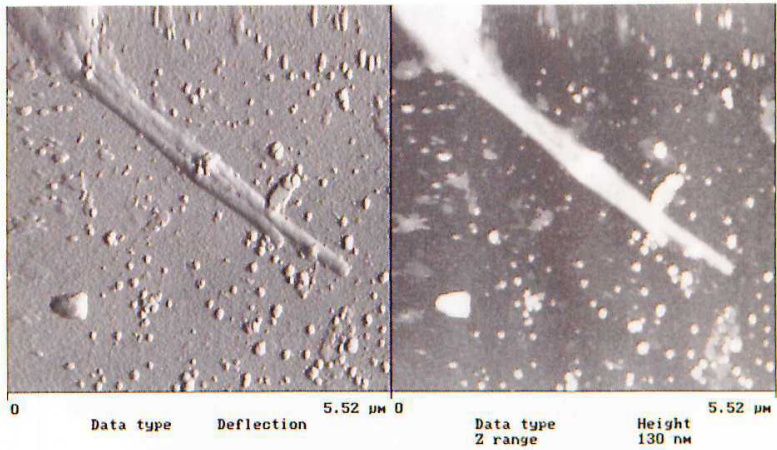

Abb. 7 (d)

schiedenen Materialien. Weitere Versuche mit Bakterienkulturen sind in Vorbereitung, um das Wachstum von Biofilmen auf unterschiedlichen Substraten besser zu verstehen und gezielt beeinflussen zu können. 


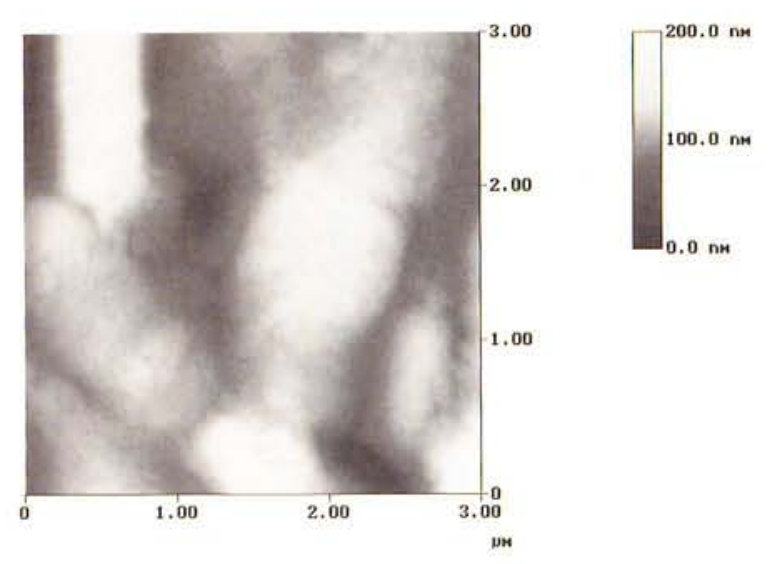

Abb. 8: SFM-Bilder von Stäbchenbakterien $(a, b)$ und von PurpurSchwefelbakterien $(c, d)$ in zwei- $(a, c)$ und dreidimensionaler Darstellung $(b, c)$.
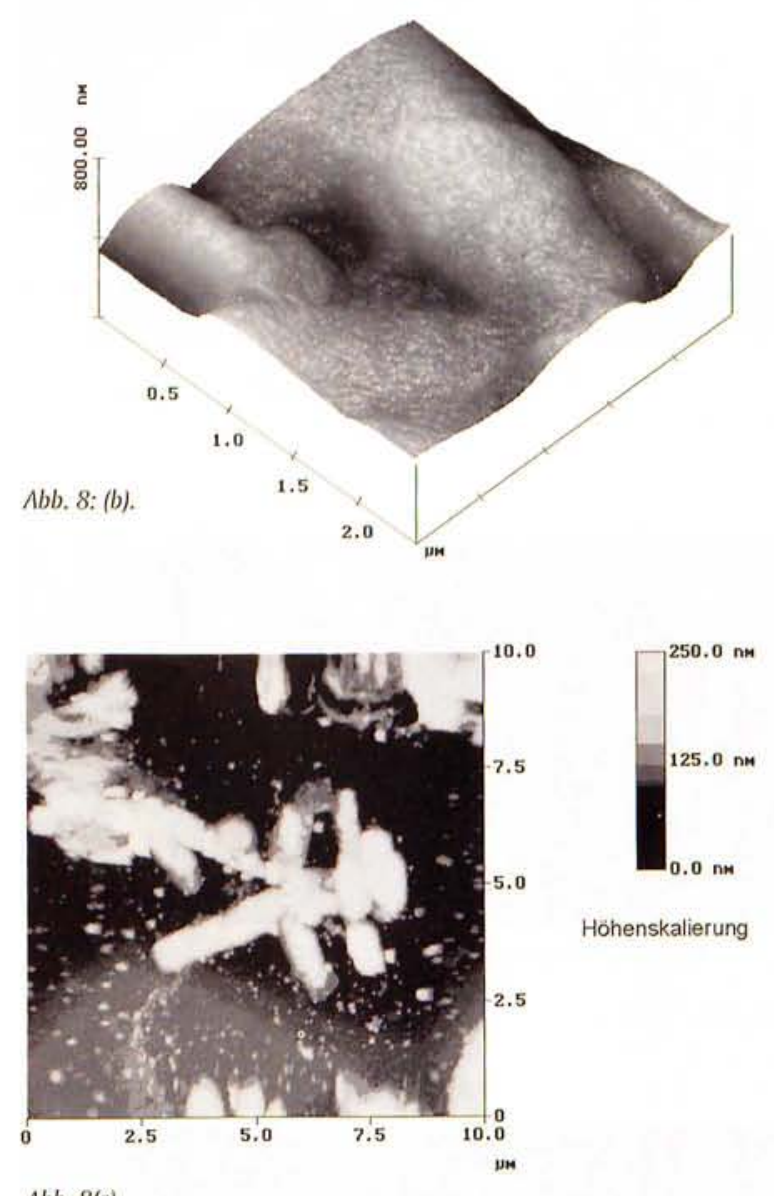

Abb. $8(c)$

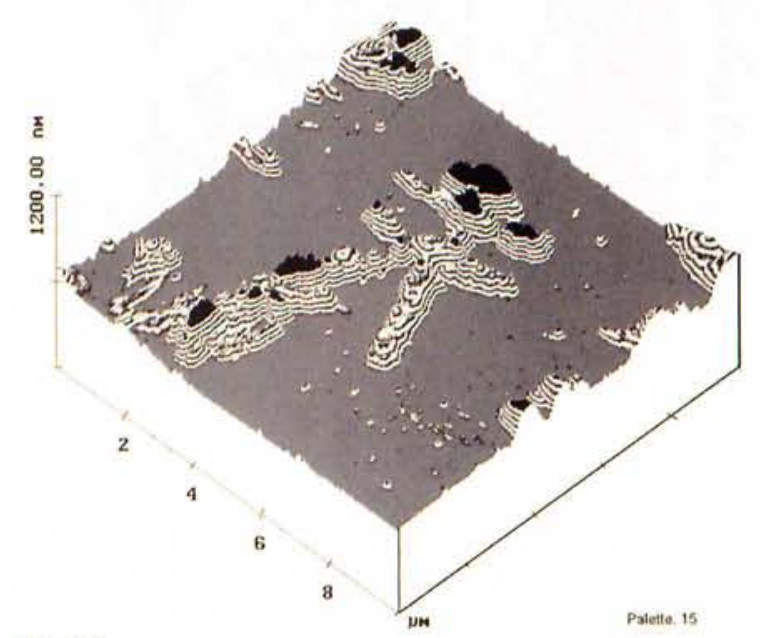

Abb. 8 (d)

\section{Literaturverzeichnis}

11/ R. Schmidt, Werkstoffverhalten in biologischen Systemen, VDI-Verlag Düsseldorf 1994.

/2/ H.C. Flemming, BIOforum 17 (1994) 61-65.

/3/ H.G. Hansma and J.H. Hoh, Annu. Rev. Biophys. Biomol. Struct. 23 (1994) 115-39.

14/ D.J. Müller, BIOspectrum 4 (1995) 30-31.

/5/ H.G. Hansma, J. Vac. Sci. Technol. B14 (1996) 13901394.

/6/ H.G. Schlegel, Allgemeine Mikrobiologie, Thieme Verlag Stuttgart 1987.

$17 /$ T.D. Brock, M.T. Madigan, J.M. Martinko, and J. Parker, Biology of Microorganisms, Prentice Hall International New York 1994.

/8/ G. Binning, H. Rohrer, Ch. Gerber, and E. Weibel, Phys. Rev. Lett. 49 (1982) 57-61.

/9/ S.N. Magonov, Appl. Spectroscopy Rev. 28 (1993).

/10/ A. Richter and R. Smith, European J. Physics 17 (1996) 311-321.

/11/ A. Richter and R. Smith, Wiss. Beiträge TFH Wildau 1 (1996) 72-78.

\section{Verfasser}

Prof. Dr. rer. nat. Asta Richter

Technische Fachhochschule Wildau

Fachbereich Ingenieurwesen/Wirtschaftsingenieurwesen Labor für Oberflächentechnik email: richter@pt.tfh-wildau.de Tel.: ++49-3375-507 219

Prof. Dr. Franz Xaver Wildenauer Technische Fachhochschule Wildau Fachbereich Ingenieurwesen/Wirtschaftsingenieurwesen Labor fuir Bioverfahrenstechnik email: wildenauer@vt.tfh-wildau.de Tel.: ++49-3375-507 227

Dipl.-Kristallograph Ronald Ries Technische Fachhochschule Wildau Fachbereich Ingenieurwesen/Wirtschaftsingenieurwesen Labor für Oberflächentechnik email: rries@pt.tfh-wildau.de Tel.: ++49-3375-507 217 